AperTO - Archivio Istituzionale Open Access dell'Università di Torino

\title{
Enhancement of mixing by rodlike polymers
}

\section{This is the author's manuscript}

Original Citation:

Availability:

This version is available http://hdl.handle.net/2318/1689602

since 2019-02-04T16:22:19Z

Published version:

DOI:10.1140/epje/i2018-11692-9

Terms of use:

Open Access

Anyone can freely access the full text of works made available as "Open Access". Works made available under a Creative Commons license can be used according to the terms and conditions of said license. Use of all other works requires consent of the right holder (author or publisher) if not exempted from copyright protection by the applicable law. 


\title{
Enhancement of mixing by rodlike polymers
}

\author{
Stefano Musacchio ${ }^{1}$, Massimo Cencini ${ }^{2}$, Emmanuel L.C. VI M. Plan ${ }^{3}$, and Dario Vincenzi ${ }^{1}$ \\ 1 Université Côte d'Azur, CNRS, LJAD, Nice, France \\ 2 Istituto dei Sistemi Complessi, CNR, via dei Taurini 19, 00185 Rome, Italy and INFN Tor Vergata \\ 3 Rudolf Peierls Centre for Theoretical Physics, University of Oxford, Oxford OX1 3PU, UK
}

Received: date / Revised version: date

\begin{abstract}
We study the mixing of a passive scalar field dispersed in a solution of rodlike polymers in two dimensions, by means of numerical simulations of a rheological model for the polymer solution. The flow is driven by a parallel sinusoidal force (Kolmogorov flow). Although the Reynolds number is lower than the critical value for inertial instabilities, the rotational dynamics of the polymers generates a chaotic flow similar to the so-called elastic-turbulence regime observed in flexible polymer solutions. The temporal decay of the variance of the scalar field and its gradients shows that this chaotic flow strongly enhances mixing.
\end{abstract}

PACS. 47.51.+a Mixing - 47.57.Ng Polymers and polymer solutions - 47.85.lk Mixing enhancement

\section{Introduction}

The mixing properties of laminar flows are generally poor. In microfluidic applications, where the Reynolds numbers are typically very low, various methods have been developed to enhance the mixing efficiency of the flow. These include the design of grooved walls, the introduction of obstacles, the use of a local forcing, or the addition of flexible polymers (e.g., Ref. [1]). In this latter case, elastic stresses can generate instabilities at vanishing fluid inertia that in turn lead to a chaotic flow known as elastic turbulence [2,3]. It was shown in Ref. [4] that a regime with features similar to those of elastic turbulence can also be obtained via the addition of rigid rodlike polymers, i.e. polymer stretching is not essential for the generation of a chaotic flow at small Reynolds numbers.

The system considered in Ref. [4] is a dilute solution of rodlike polymers driven by a sinusoidal parallel body force (the Kolmogorov force) at a Reynolds number lower than the critical value for inertial hydrodynamic instabilities. A similar setting was used previously to study elastic turbulence induced by flexible polymer solutions [5]. For low rodlike polymer concentrations, the flow is laminar and only displays small deviations from the Newtonian regime. However, when the concentration is increased beyond a critical value, the flow becomes chaotic, the streamlines oscillate and thin vorticity filaments form. An inspection of the snapshots of the vorticity and polymer-orientation fields show that perturbations in the flow are associated with strong deviations of the polymer orientation from the mean-flow direction. The kinetic energy fluctuates around a stationary value lower than that of the laminar case and the mean power required to maintain the mean flow grows with the concentration, which signals a corresponding increase of the kinetic-energy dissipation. In this regime, the Reynolds stress is negligible compared to the polymer and viscous ones. In particular, the polymer stress increases as a function of polymer concentration. Thus, the chaotic dynamics is entirely due to the rotation of polymers, while fluid inertia plays no role. This is further confirmed by the analyis of the kinetic-energy balance in Fourier space. The nonlinear coupling between different Fourier modes due to inertia is indeed negligible; the dynamics of the flow rather results from a scale-by-scale balance between polymer transfer and viscous dissipation. Finally, the kineticenergy spectrum displays a power law $k^{-\alpha}$ with $\alpha \leqslant 3$, where $k$ is the wave number. A large number of Fourier modes are thus excited, but the energy is concentrated on the large scales and fluctuations decay rapidly with the wave number. It should be noted that the exponent $\alpha$ is not universal, since it depends on polymer concentration and the details of the forcing.

Even though the chaotic regime described above is not generated by polymer stretching, it is similar to elastic turbulence in solutions of flexible polymers. The goal of this paper is to show that the addition of rodlike polymers can be effectively used to enhance mixing at small Reynolds numbers.

\section{Passive-scalar dispersion in a solution of rodlike polymers}

The mixing efficiency of a flow can be quantified by studying its ability to disperse a passive scalar field, such as a colorant injected in the fluid. 


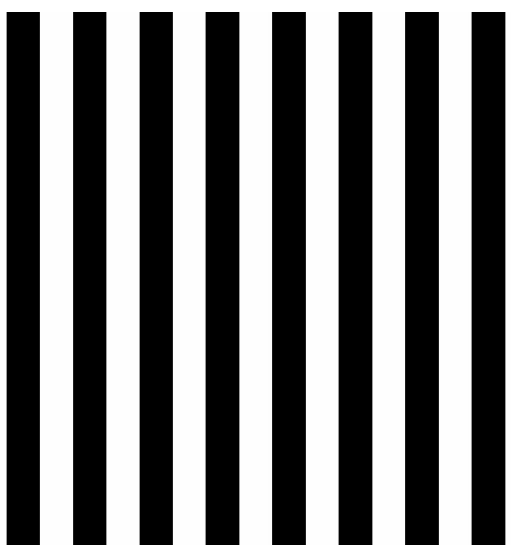

(a1) $\eta_{p}=0, t=0$

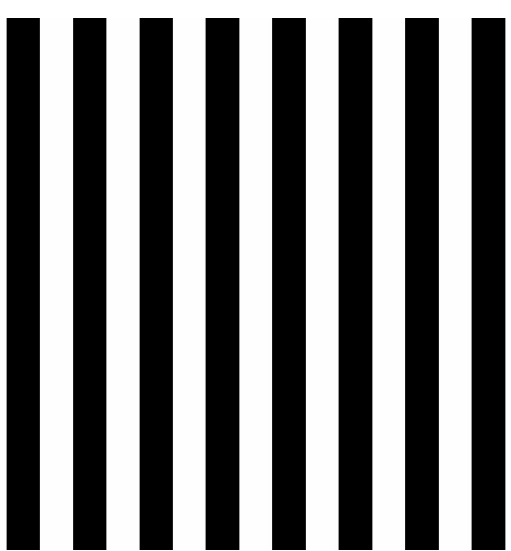

(b2) $\eta_{p}=5, t=0$

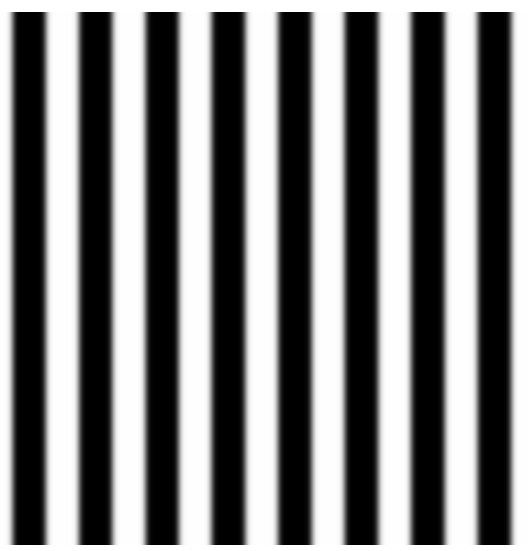

(a2) $\eta_{p}=0, t=0.6$

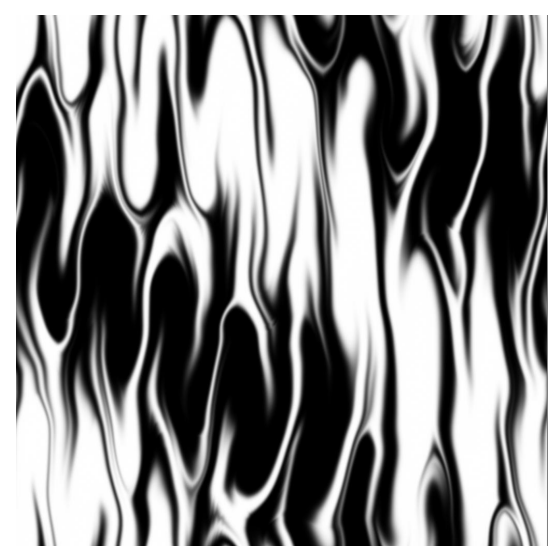

(b3) $\eta_{p}=5, t=0.6$

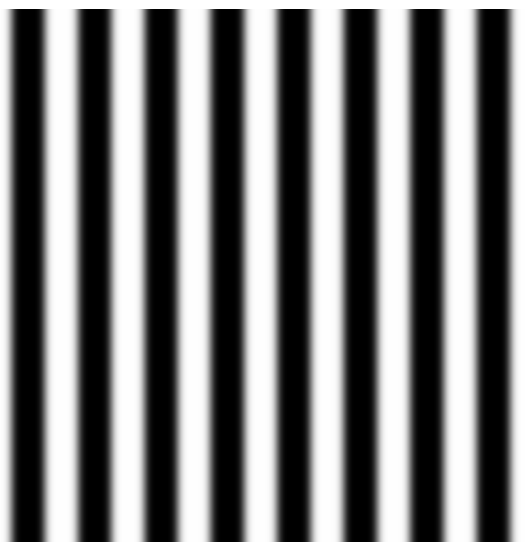

(a3) $\eta_{p}=0, t=1.2$

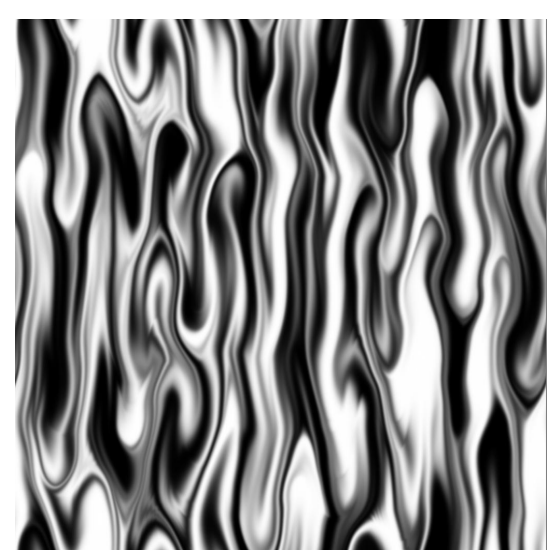

(b4) $\eta_{p}=5, t=1.2$

Fig. 1: Snapshots of the passive scalar field $\theta(\boldsymbol{x}, t)$ at $t=0,0.6,1.2$ (from left to right) for $\eta_{p}=0$ (top) and $\eta_{p}=5$ (bottom) and initial condition B. Here $\mathrm{Pe}=1000$.

We consider a scalar field $\theta(\boldsymbol{x}, t)$ with diffusivity $D$ in a two-dimensional solution of rodlike polymers. The dynamics of $\theta$ is ruled by the advection-diffusion equation

$$
\partial_{t} \theta+\boldsymbol{u} \cdot \nabla \theta=D \Delta \theta
$$

with $\boldsymbol{u}(\boldsymbol{x}, t)$ being the incompressible velocity field of the solution. The polymer phase is described by the unittrace symmetric tensor field $\mathrm{R}(\boldsymbol{x}, t)$, the first eigenvector of which yields the average orientation of polymers in a volume element centred at $\boldsymbol{x}$ at time $t$. In Doi and Edwards' decoupling approximation the fluid and polymer phases evolve according to the following equations [6]:

$$
\begin{aligned}
\partial_{t} \boldsymbol{u}+\boldsymbol{u} \cdot \nabla \boldsymbol{u}= & -\nabla p+\left(\nu+\nu_{p}\right) \Delta \boldsymbol{u} \\
& +6 \nu \eta_{p} \nabla \cdot[(\nabla \boldsymbol{u}: \mathrm{R}) \mathrm{R}]+\boldsymbol{f} \\
\partial_{t} \mathrm{R}+\boldsymbol{u} \cdot \nabla \mathrm{R}= & (\nabla \boldsymbol{u}) \mathrm{R}+\mathrm{R}(\nabla \boldsymbol{u})^{\top} \\
& -2(\nabla \boldsymbol{u}: \mathrm{R}) \mathrm{R}-2 \alpha(2 \mathrm{R}-\mathrm{I}),
\end{aligned}
$$

where $(\nabla \boldsymbol{u})_{i j}=\partial u_{i} / \partial x_{j}, p$ is pressure, $\nu$ is the kinematic viscosity of the solvent, $\mathrm{I}$ is the identity matrix, and $\nu_{p}$ and $\alpha$ are proportional to the orientational diffusivity of polymers. In numerical simulations a diffusive term $\kappa \Delta \mathrm{R}$ (we used $\kappa=3 \times 10^{-3}$ ) is added to Eq. (3) in order to improve stability [7]. The parameter $\eta_{p}$ determines the coupling between the polymer phase and the fluid and is an increasing function of the polymer concentration. The values of $\eta_{p}$ considered here $\left(\eta_{p} \leqslant 5\right)$ correspond to a dilute solution $[4,8]$. The above polymer model was studied extensively in the turbulent-drag-reduction regime at high Reynolds number [9].

The system is driven by the Kolmogorov force $\boldsymbol{f}(\boldsymbol{x})=$ $(0, F \sin (K x))$, where $F$ and $K$ are the amplitude and the wave number of the force, respectively. In the Newtonian case $\left(\eta_{p}=0\right)$, the Navier-Stokes equations admit the laminar solution $\boldsymbol{u}(\boldsymbol{x})=\left(0, U_{0} \sin (K x)\right)$ with $U_{0}=$ $F / \nu K^{2}$. This solution is stable if the Reynolds number $\operatorname{Re}=U_{0} / \nu K$ is smaller than the critical value $\operatorname{Re}_{c}=\sqrt{2}$ (e.g., Ref. [10]). In the following, we take $\mathrm{Re}=1<\mathrm{Re}_{c}$ in order to ensure that inertial effects are negligible and that the chaotic regime arises solely from the rotational dynamics of the rodlike polymers. 


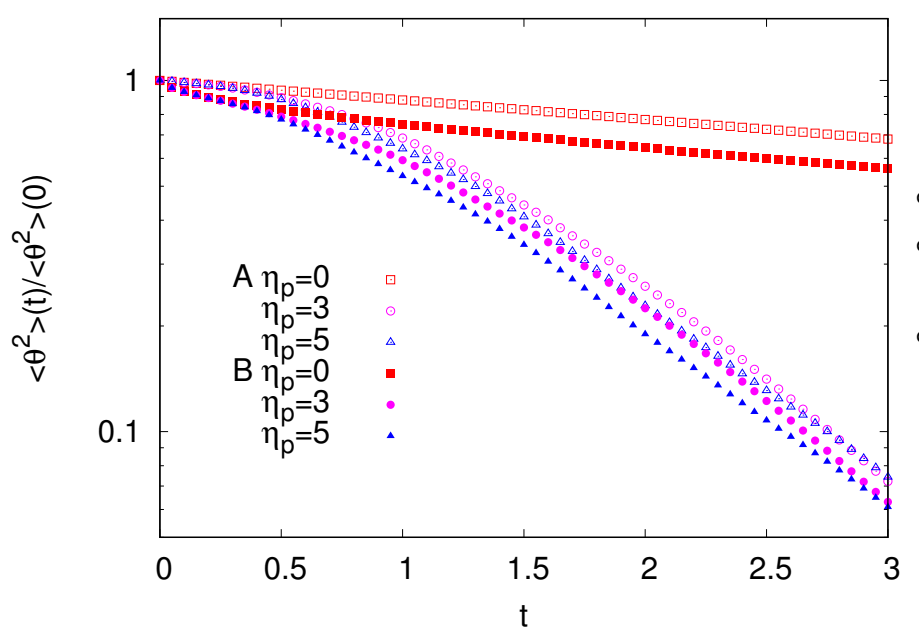

Fig. 2: Temporal decay of the variance $\left\langle\theta^{2}\right\rangle(t)$, normalized with its initial value at $t=0$, for $\eta_{p}=0$ (squares), $\eta_{p}=3$ (circles), $\eta_{p}=5$ (triangles), and for initial conditions $\mathrm{A}$ (empty symbols) and B (filled symbols). Here $\mathrm{Pe}=1000$.

Equations (1), (2) and (3) are solved on a $2 \pi \times 2 \pi$ - domain periodic in both directions by using a $1 / 2$-dealiased pseudospectral method on a grid with $1024^{2}$ mesh points. Time integration is performed via a fourth-order RungeKutta scheme. In the numerical simulations presented below, we use $K=8, F=512$ and $\nu=1$. The molecular diffusivity is $D=10^{-3}$ for most of the simulations reported in Sect. 3. The corresponding Péclet number $\mathrm{Pe}=U_{0} / K D$ is $\mathrm{Pe}=1000$. For the study of the Péclet number effects we also consider $D=5 \times 10^{-4}(\mathrm{Pe}=2000)$ and $D=2.5 \times 10^{-4}$ $(\mathrm{Pe}=4000)$. In addition, the orientational diffusion of polymers is disregarded (i.e., we take $\nu_{p}=0$ and $\alpha=0$ ) for two reasons: 1 ) it is expected to play a minor role in the chaotic regime and 2) we wish to ensure that the chaotic regime is not triggered by Brownian fluctuations.

In the Newtonian case $\left(\eta_{p}=0\right)$, the dynamics of the velocity field is independent of $\mathrm{R}$ and the laminar solution is stable at $\mathrm{Re}=1$. Therefore we simply integrate Eq. (1) with $\boldsymbol{u}(\boldsymbol{x})=\left(0, U_{0} \sin (K x)\right)$. Conversely, in the non-Newtonian case $\left(\eta_{p}>0\right)$, we performed a preliminary set of simulations by integrating Eqs. (2) and (3) with initial condition for the velocity field obtained as a small perturbation of the Newtonian stable flow and with the components of $\mathrm{R}$ initially distributed randomly. Once the flow has reached the statistically stationary chaotic regime, we start to integrate the dynamics of the scalar field $\theta$.

The initial condition for the scalar field, $\theta(\boldsymbol{x}, 0)$, is taken independent of $y$, so that the initial scalar gradient $\nabla \theta$ is oriented in the $x$ direction, i.e, perpendicular to the direction of the laminar flow for $\eta_{p}=0$. This choice ensures that the mixing in the absence of polymers is solely due to molecular diffusion. Two different initial conditions are considered: A) the monocromatic function $\theta(\boldsymbol{x})=$ $\cos (K x)$ and B) the step function $\theta(\boldsymbol{x})=\operatorname{sign}[\cos (K x)]$. In both cases we fix $K=8$, i.e., the same wavenumber

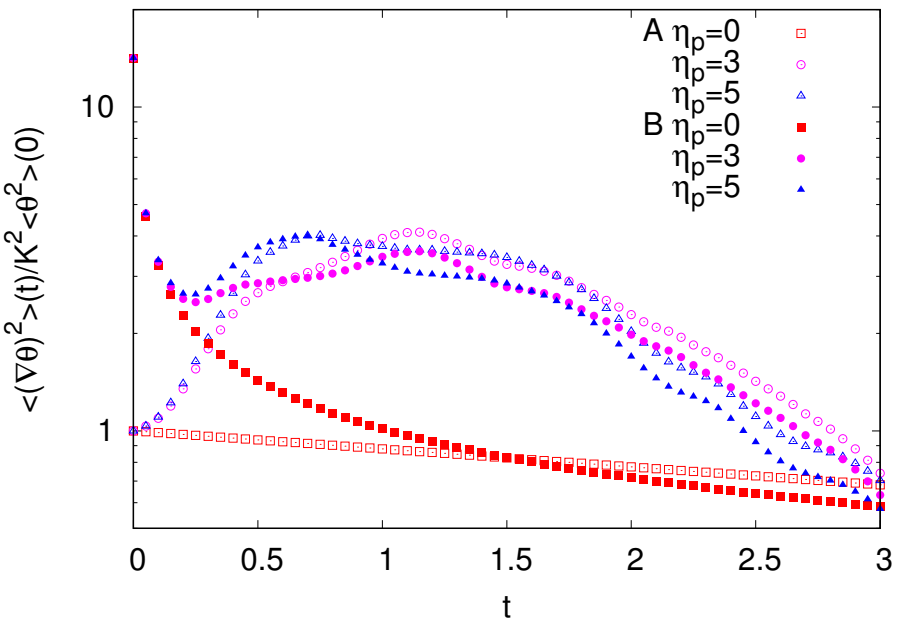

Fig. 3: Temporal evolution of the variance of the scalar gradients, $\left\langle(\nabla \theta)^{2}\right\rangle(t)$, for $\eta_{p}=0$ (squares), $\eta_{p}=3$ (circles), $\eta_{p}=5$ (triangles), and for initial conditions $\mathrm{A}$ (empty symbols) and B (filled symbols). Here Pe $=1000$.

of the base flow. For the former initial condition and in the Newtonian case $\left(\eta_{p}=0\right)$, the exponential decay rate of the scalar variance $\left\langle\theta^{2}\right\rangle(t) \equiv \int \theta^{2}(\boldsymbol{x}, t) \mathrm{d} \boldsymbol{x}$ is known analytically as $\beta_{0}=-\mathrm{d} \log \left[\left\langle\theta^{2}\right\rangle(t)\right] / \mathrm{d} t=2 D K^{2}$. The latter initial condition is chosen to mimic the experimental setting in which two differently coloured fluids are injected in a microchannel [3].

\section{Mixing enhancement}

In Figure 1 we compare the temporal evolution of the scalar field with and without polymers starting from initial condition B. In the absence of polymers, molecular diffusion simply blurs the borders between the white and black stripes, and even after a long time the scalar field remains essentially unmixed. Conversely, over a comparable time interval the chaotic flow induced by the rodlike polymers mixes the scalar field efficiently.

To quantify the gain in mixing efficiency, we study the temporal behaviour of the variance of the scalar field and of its gradients. As shown in Fig 2, after an initial transient, the decay rate of $\left\langle\theta^{2}\right\rangle$ becomes independent of the specific choice of the initial condition. In the Newtonian case, we recover the analytical prediction $\left\langle\theta^{2}\right\rangle(t) \propto$ $\exp \left(-\beta_{0} t\right)$. For $\eta_{p}>0$, we find that the decay is much faster. The similar decay observed for the cases $\eta_{p}=3$ and $\eta_{p}=5$ suggests a weak dependence of the decay rate on the concentration of polymers. In Ref. [4], it was found that increasing $\eta_{p}$ at fixed forcing amplitude $F$ the resulting chaotic flow displays stronger fluctuations, but the amplitude of the mean flow (which remains sinusoidal) is reduced. Likely, the combined effect of the reduction of the mean flow and the growth of the fluctuations, results in a comparable mixing efficiency for the two cases considered here. 


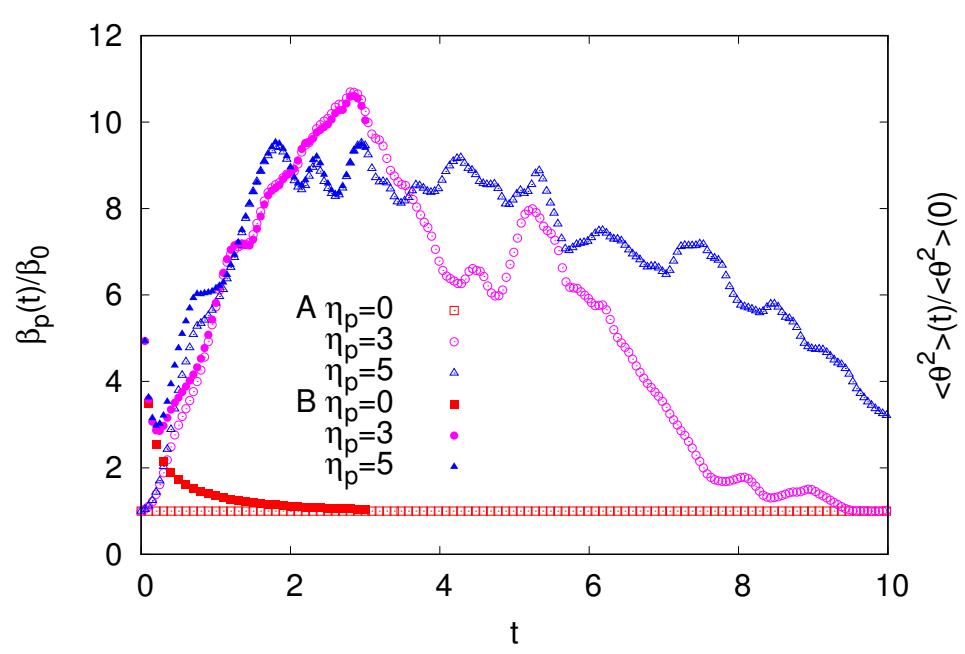

Fig. 4: Instantaneous exponential decay rate $\beta_{p}(t)$, normalized with $\beta_{0}=2 D K^{2}$, for $\eta_{p}=0$ (squares), $\eta_{p}=3$ (circles), $\eta_{p}=5$ (triangles), and for initial conditions $\mathrm{A}$ (empty symbols) and B (filled symbols). Here $\mathrm{Pe}=1000$.

The time behaviour of $\left\langle\theta^{2}\right\rangle$ is closely related to that of the scalar gradients. In the absence of polymers, $\left\langle(\nabla \theta)^{2}\right\rangle$ asymptotically decays with the same rate as the variance of the field. At short times, however, the decay of $\left\langle(\nabla \theta)^{2}\right\rangle$ depends on the initial condition (Fig. 3). In case A, which is monochromatic, the decay is purely exponential from the beginning, whereas in case B the decay is faster, since each Fourier mode $k$ of the scalar field decays with a different exponential rate $-2 D k^{2}$. In the presence of polymers and in case $\mathrm{A},\left\langle(\nabla \theta)^{2}\right\rangle$ initially grows because mixing creates thin scalar filaments (the so-called direct cascade of passive scalars); at later times when the gradient scale reaches the diffusive scale we observe a rapid decay, with a rate similar to that of $\left\langle\theta^{2}\right\rangle$, which indicates that the increased mixing efficiency of the polymer solution with respect to the Newtonian fluid. Case B is similar to case A, except for an initial transient characterized by the fast diffusive decay of the high Fourier modes of the initial condition.

To accurately measure the asymptotic decay of the scalar variance, it is useful to introduce the instantaneous exponential decay rate

$$
\beta_{p}(t)=-\frac{\mathrm{d}}{\mathrm{d} t} \log \left\langle\theta^{2}\right\rangle=\beta_{0} \frac{\left\langle(\nabla \theta)^{2}\right\rangle}{K^{2}\left\langle\theta^{2}\right\rangle} .
$$

The ratio $\beta_{p}(t) / \beta_{0}$ quantifies the increase of the mixing efficiency due to the addition of polymers with respect to molecular diffusion only. As shown in Fig 4, the two initial conditions $\mathrm{A}$ and $\mathrm{B}$ recover the same values of $\beta_{p}(t)$ after an initial transient. For $\eta_{p}=3$ we observe initially a rapid increase of $\beta_{p}(t)$, which reaches values much larger than $\beta_{0}$. However, at long times, when the scalar field is almost completely homogeneized, $\beta_{p}(t)$ reduces and eventually returns close to $\beta_{0}$. For $\eta_{p}=5$, after an initial growth similar to the $\eta_{p}=3$ case, $\beta_{p}(t)$ seems to fluctuate around a constant mean value $\beta_{*}$ in the time interval

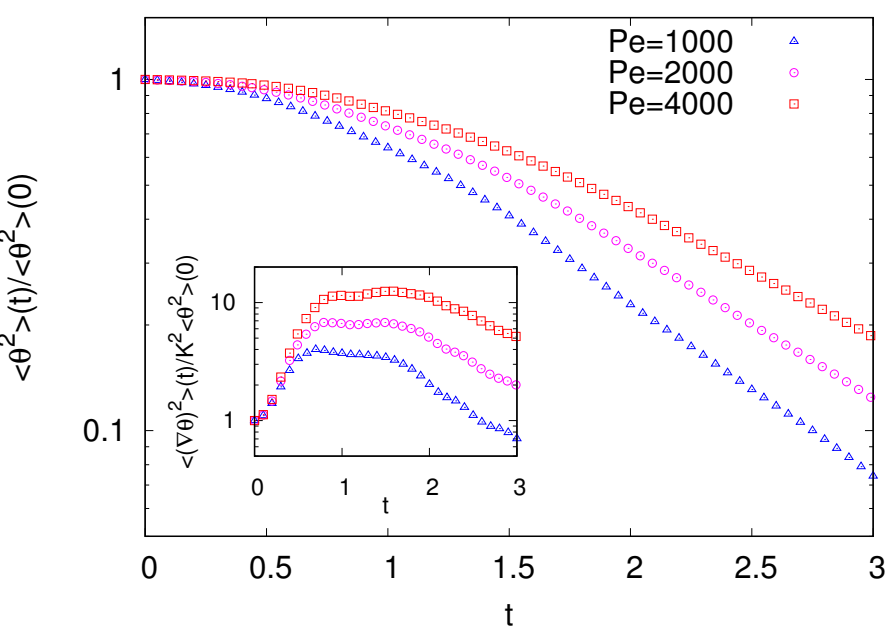

Fig. 5: Temporal evolution of the variance $\left\langle\theta^{2}\right\rangle(t)$ for $P e=1000$ (triangles), $P e=2000$ (circles) and $P e=$ 4000 (squares). Here $\eta_{p}=5$ and the initial condition is monochromatic (case A). Inset: Temporal evolution of the variance of the scalar gradients $\left\langle(\nabla \theta)^{2}\right\rangle(t)$.

$t \in[2,4]$. At later times $\beta_{p}(t)$ decreases, but its decay is slower than for $\eta_{p}=3$.

We notice that an exponential decay of the passive scalar variance with a constant rate $\beta_{p}(t)$ implies that $\left\langle(\nabla \theta)^{2}\right\rangle$ must become proportional to $\left\langle\theta^{2}\right\rangle$, meaning that the typical scale of the scalar gradients, defined as $\ell=$ $\left[\left\langle\theta^{2}\right\rangle /\left\langle(\nabla \theta)^{2}\right\rangle\right]^{1 / 2}$, remains constant. For $\eta_{p}=5$ we have that the scale separation between the large scale of the base flow $L_{u}=2 \pi / K$ and $\ell$ is $L_{u} / \ell \simeq 3$ for $t \in[2,4]$.

Theoretical predictions on the asymptotic decay of the scalar field have been derived by exploiting the relation between the statistics of Lagrangian trajectories and the statistics of the passive scalar $[11,12]$. In particular, it has been shown that for smooth, statistically homogeneous and isotropic flows, in the limit $\mathrm{Pe} \rightarrow \infty$ and for large times, the moments of the passive scalar decays exponentially $\left\langle|\theta|^{n}\right\rangle \propto \exp \left(-\gamma_{n} t\right)$, with $\gamma_{n}$ linked to the stretching rate statistics. In our notation $\beta$ corresponds to $\gamma_{2}$. The fact that we observe an exponential decay with constant $\beta$ only for intermediate times could be due to various causes. The mechanism for the exponential decay [11] originates from the chaotic stretching of the passive scalar, which is effective when there is a large scale separation between the typical scale of the flow $L_{u}$ and the diffusive scale (i.e., in the limit $\mathrm{Pe} \rightarrow \infty)$. In the following we will show that the asymptotic decrease of $\beta_{p}(t)$ observed in Fig 4 could be related to the finite values of $D$ required by the numerical simulations. Further, the Kolmogorov flow is neither homogeneous nor isotropic. Moreover, in our case the characteristic large scale of the passive scalar $L_{\theta}=2 \pi / K$ is equal to that of the base flow $L_{u}$. When $L_{\theta} \sim L_{u}$ it has been shown that the decay, though still exponential, is dominated by "strange eigenmodes" of the advectiondiffusion operator [13], and its connection to Lagrangian stretching rate become more complex [12]. 


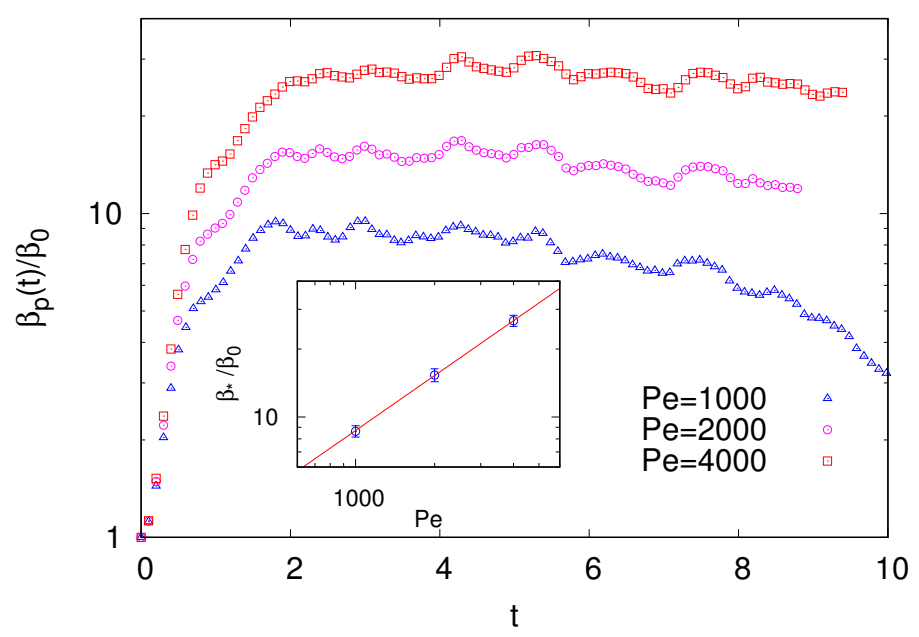

Fig. 6: Instantaneous exponential decay rate $\beta_{p}(t)$, normalized with $\beta_{0}=2 D K^{2}$, for $\mathrm{Pe}=1000$ (triangles), $\mathrm{Pe}=2000$ (circles) and $\mathrm{Pe}=4000$ (squares). Here $\eta_{p}=5$ and the initial condition is monochromatic (case A). Inset: Average exponential decay rate $\beta_{*}$ as a function of $\mathrm{Pe}$ (circles). The line represents the scaling $P e^{0.8}$.

On the basis of the previous observations, we expect that the gain in mixing efficiency due to the addition of polymers should depend on the Péclet number $\mathrm{Pe}=$ $U_{0} / K D$. Increasing Pe at fixed Re reduces $\beta_{0}$ but increases the generation of smaller scales in the scalar field, thus leading to higher values of $\left\langle(\nabla \theta)^{2}\right\rangle$.

In order to investigate the dependence on Pe, we have compared three simulations with initial condition $\mathrm{A}$ at $\eta_{p}=5$ and $\operatorname{Re}=1$ with different diffusivities: $D=10^{-3}$ $(\mathrm{Pe}=1000) D=5 \times 10^{-4}(\mathrm{Pe}=2000)$ and $D=2.5 \times$ $10^{-4}(\mathrm{Pe}=4000)$. The decay of the variance for the three cases is shown in Fig. 5. Although an increase of $\left\langle(\nabla \theta)^{2}\right\rangle$ is observed as a function of $\mathrm{Pe}$ at all times (see inset of Fig. 5), the decay of $\left\langle\theta^{2}\right\rangle$ is slower at greater Pe because $\beta_{0}$ is reduced. Nevertheless, Fig. 6 shows that the gain in mixing efficiency increases with Pe. A power-law fit of $\beta_{*}$, obtained by averaging $\beta_{p}(t) / \beta_{0}$ in the interval $2.5<$ $t<4$, indicates a growth proportional to $\mathrm{Pe}^{0.8}$. At present we do not have a clear understanding of this power law dependence. We notice that at increasing Pe, the regime in which $\beta_{p}(t)$ is almost constant continues for longer times. This suggests that the asympotic decay of $\beta_{p}(t)$ might be a finite-Pe effect.

\section{Conclusions}

The addition of rodlike polymers to a low-Reynolds-number Newtonian fluid generates a chaotic flow, similarly to the elastic turbulence regime observed in flexible polymers solutions. We have shown that this regime strongly enhances the mixing of a passive scalar dispersed in the solution. In particular, the variance of the scalar field and of its gradients decays much faster than in the purely dif- fusive case. Moreover, we found that this effect increases with the Péclet number. In order to quantify the gain in the mixing efficiency we introduced the instantaneous exponential decay rate $\beta_{p}(t)$. The rapid initial growth of $\beta_{p}(t)$ to values much higer than the diffusive decay rate $\beta_{0}$ provides a precise measure of the increased mixing. Our results also show that for high Péclet number and high polymer concentrations, the decay of the scalar variance displays an almost exponential regime, in which $\beta_{p}(t)$ fluctuates around a constant mean value.

Although our study is conducted in an idealized setting, we hope that it can motivate experimental investigations of the gain in mixing efficiency obtained via the addition of rodlike polymers to a Newtonian fluid at low Reynolds number. In future investigations it would also be interesting to compare the gain in mixing efficiency obtained with rodlike or flexible polymer solutions at similar concentrations.

\section{Acknowledgments}

The authors acknowledge the support of the EU COST Action MP 1305 "Flowing Matter". M.C. was partially supported by Université Côte d'Azur through Investissements d'Avenir UCA ref. ANR-15-IDEX-01.

\section{Authors contributions}

All the authors were involved in the preparation of the manuscript. All the authors have read and approved the final manuscript.

\section{References}

1. T.M. Squires, S.R. Quake, Rev. Mod. Phys. 77, 977 (2005).

2. A. Groisman, V. Steinberg, Nature 405, 53 (2000).

3. A. Groisman, V. Steinberg, Nature 410, 905 (2001).

4. E.L.C. VI M. Plan, S. Musacchio, D. Vincenzi, Phys. Rev. E 96, 053108 (2017).

5. S. Berti, A. Bistagnino, G. Boffetta, A. Celani, and S. Musacchio. Phys. Rev. E 77, 055306 (2008).

6. M. Doi, S.F. Edwards, The Theory of Polymer Dynamics (Oxford University Press, Oxford, 1988).

7. R. Sureshkumar, A.N. Beris, J. Non-Newtonian Fluid Mech. 60, 53 (1995).

8. Y. Amarouchene, D. Bonn, H. Kellay, T.S. Lo, V.S. L'vov, I. Procaccia, Phys. Fluids 20, 065108 (2008).

9. I. Procaccia, V.S. L'vov, R. Benzi, Rev. Mod. Phys. 80, 225 (2008).

10. S. Musacchio, G. Boffetta, Phys. Rev. E 89, 023004 (2014).

11. E. Balkovsky, A. Fouxon, Phys, Rev. E 60, 4164 (1999).

12. P.H. Haynes, J. Vanneste, Phys. Fluids 17, 097103 (2005).

13. J. Sukhatme, R.T. Pierrehumbert, Phys. Rev. E 66, 056302 (2002). 